Journal of

Materials Chemistry B

Materials for biology and medicine

www.rsc.org/MaterialsB

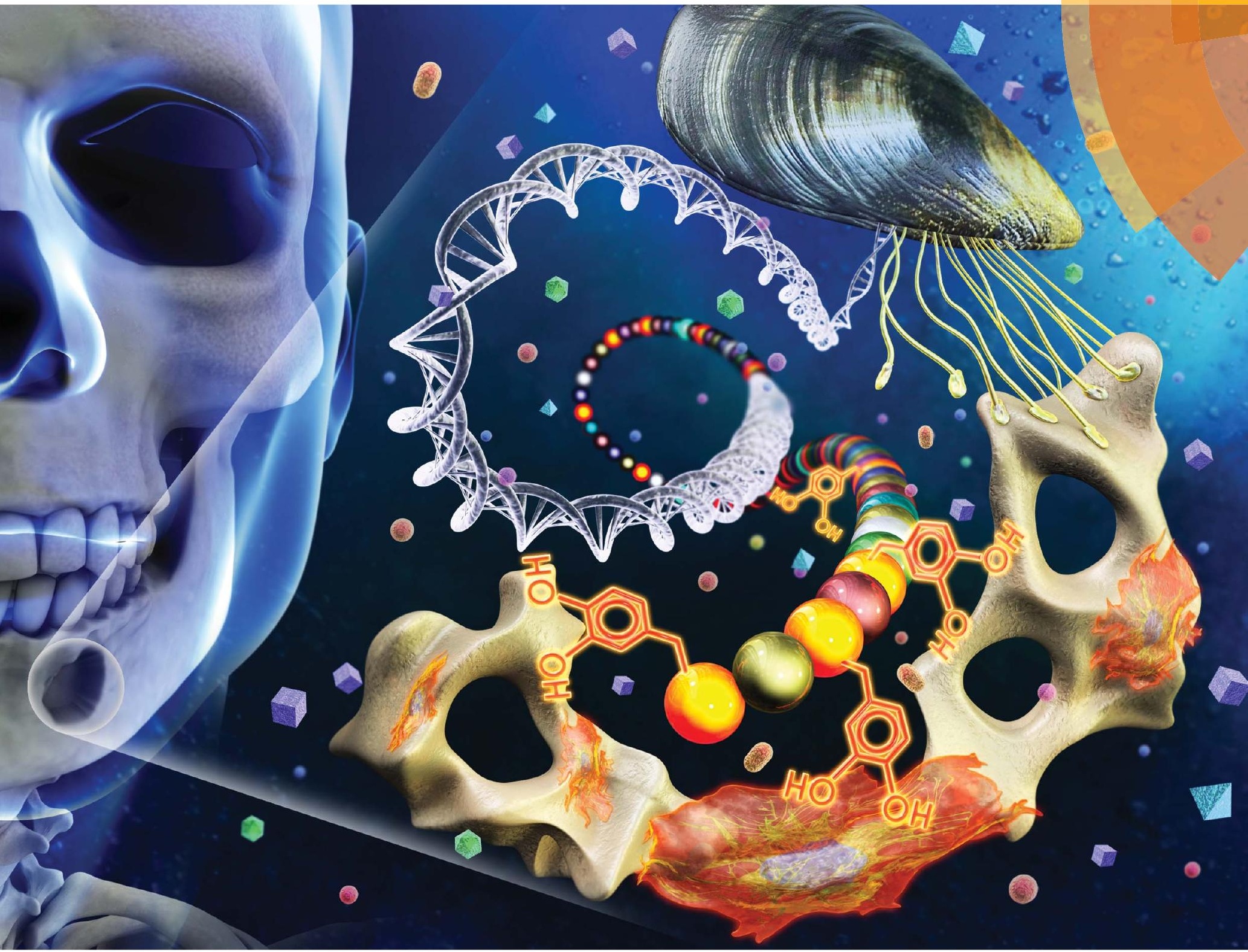

ISSN 2050-750X

\title{
PAPER
}

Hyung Joon Cha, Sang Ho Jun et al.

Engineered mussel bioglue as a functional osteoinductive binder

for grafting of bone substitute particles to accelerate in vivo bone 


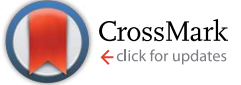

Cite this: J. Mater. Chem. B, 2015, 3, 546

Received 20th July 2014

Accepted 25th November 2014

DOI: 10.1039/c4tb01197j

www.rsc.org/MaterialsB

\section{Engineered mussel bioglue as a functional osteoinductive binder for grafting of bone substitute particles to accelerate in vivo bone regeneration}

\author{
Bong-Hyuk Choi, ${ }^{a}$ Hogyun Cheong, ${ }^{a}$ Jin-Soo Ahn, ${ }^{b}$ Cong Zhou, ${ }^{c}$ Jong Jin Kwon, ${ }^{d}$ \\ Hyung Joon Cha*a and Sang Ho Jun*d
}

\begin{abstract}
Xenograft bone substitutes, such as deproteinized bovine bone mineral (DBBM), have been widely employed as osteoconductive structural materials for bone tissue engineering. However, the loss of xenograft bone substitute particles in defects has been a major limitation, along with a lack of osteoinductive function. Mussel adhesive protein (MAP), a remarkable and powerful adhesive biomaterial in nature, can attach to various substrates, even in wet environments. Its adhesive and water-resistant abilities are considered to be mainly derived from the reduced catechol form, 3,4dihydroxyphenylalanine (DOPA), of its tyrosine residues. Here, we evaluated the use of DOPA-containing MAP as a functional binder biomaterial to effectively retain DBBM particles at the defect site during in vivo bone regeneration. We observed that DOPA-containing MAP was able to bind DBBM particles easily to make an aggregate, and grafted DBBM particles were not lost in a defect in the rat calvaria during the healing period. Importantly, grafting of a DOPA-containing MAP-bound DBBM aggregate resulted in remarkably accelerated in vivo bone regeneration and even bone remodeling. Interestingly, we found that the DOPA residues in the modified MAP had an osteoinductive ability based on clear observation of the in vivo maturation of new bones with a similar bone density to the normal bone and of the in vitro osteogenic differentiation of osteoblast cells. Collectively, DOPA-containing MAP is a promising functional binder biomaterial for xenograft bone substitute-assisted bone regeneration with enhanced osteoconductivity and acquired osteoinductivity. This mussel glue could also be successfully utilized as a potential biomaterial for general bone tissue engineering.
\end{abstract}

\section{Introduction}

Bone is a specialized tissue that serves multiple functions, such as structural support, protection of vital organs, and metabolic regulation of mineral balance. ${ }^{1}$ Thus, effective treatment or replacement of osseous defects is important in bone tissue engineering. ${ }^{2}$ Autologous grafts, usually harvested from a patient's own bone, are the ideal materials for bone substitutes because of their osteogenic, osteoinductive, and osteoconductive properties. ${ }^{3}$ However, there are many barriers to the use of autologous grafts, such as limited availability, requirement of a second surgery, increased operation time, lack of graft quantity, secondary infection or bleeding, chronic donor site

${ }^{a}$ Department of Chemical Engineering, Pohang University of Science and Technology, Pohang 790-784, Korea. E-mail: hjcha@postech.ac.kr

${ }^{b}$ Dental Research Institute and Department of Dental Biomaterials Science, Seoul National University, Seoul 110-749, Korea

${ }^{c}$ Department of Medicine, Korea University Graduate School, Seoul 136-705, Korea

${ }^{d}$ Division of Oral and Maxillofacial Surgery, Department of Dentistry, Anam Hospital, Korea University Medical Center, Seoul 136-705, Korea.E-mail: junsang@korea.ac.kr pain, and additional preparation cost. ${ }^{3}$ To overcome these disadvantages of autologous grafts, several bone graft substitutes, such as allografts, xenografts, and alloplasts, are used as filling materials because of their compositional similarity, biocompatibility, and osteoconductivity. ${ }^{4-6}$ Among these bone substitutes, xenografts are commonly used in all fields of orthopedic and dental surgery, based on much evidence for their suitability in in vivo applications.

Clinically, xenograft bone substitutes, such as deproteinized bovine bone mineral (DBBM), are typically used in a hydrated form, mixed with saline or the patient's blood, although these substitute particles are not well agglomerated. Because xenograft bone substitute particles are not well retained at the defect site, their displacement reduces the osteoconductivity before bone formation is properly generated. To date, diverse approaches have been attempted to solve these problems caused by using xenograft particles. ${ }^{7-12}$ One major approach is to employ a physical barrier using non-resorbable materials (e.g., titanium mesh and expanded polytetrafluorethylene) or resorbable materials (e.g., collagen membrane, polylactic acid, 
polyglycolic acid, and polyurethane). ${ }^{7-10}$ Although non-resorbable barrier membranes provide an effective barrier function for a sufficient period of time, they require a second surgery for their removal. ${ }^{7,9}$ In contrast, resorbable barrier membranes can be resorbed by the body; however, these membranes have several weaknesses, such as unpredictable degradation time and weak rigidity. ${ }^{7}$ Another approach is mixing with sticky biomaterials, such as collagen and hyaluronic acid, without a barrier membrane. ${ }^{11,12}$ Several reports have provided evidence for improved bone regeneration by increasing the retention of xenograft particles by using sticky materials. However, these sticky materials usually lack osteoinductivity and thus should be further investigated to prove their efficacy.

Mussel adhesive proteins (MAPs) have been paid great attention as biomaterials in tissue and biomedical engineering because of their superior properties, including biocompatibility and surface-independent adhesiveness, even in a wet environment. $^{13,14}$ Previously, recombinant hybrid-type MAP was designed and mass-produced in Escherichia coli to overcome the limited availability of natural MAPs. ${ }^{15}$ This hybrid MAP is composed of six repeats of type 1 protein (fp-1) decapeptide at both the $\mathrm{N}$ - and the C-termini of type 5 protein (fp-5). In general, the outstanding adhesive properties of MAPs are attributed to 3,4-dihydroxyphenylalanine (DOPA), which is the hydroxylated form of tyrosine. ${ }^{16}$ Aside from its adhesive role as a protein constituent, several reports show that the DOPA molecule shows osteoinductive activity in vitro ${ }^{17-19}$ and in vivo. ${ }^{20}$

In the present work, we evaluated the in vivo potential of MAP as a functional osteoinductive binder material for bone regeneration in bone tissue engineering. We confirmed both the binding of DOPA-containing MAP ( $m$ MAP) to DBBM particles and the effects of mMAP-bound DBBM on new bone formation in a defect in the rat calvaria. We also investigated several in vitro cellular behaviors, such as adhesion, proliferation, spreading, and differentiation, on an mMAP-coated surface to assess the osteoinductive ability of $m$ MAP.

\section{Experimental}

\section{Preparation of $m$ MAP}

The expression and purification of a recombinant hybrid-type MAP, fp-151, were performed as described in previous studies. ${ }^{15,21-23}$ For in vitro DOPA modification, $2 \mathrm{~g} \mathrm{~L}^{-1}$ of purified original MAP was dissolved in $200 \mathrm{mM}$ acetate buffer (pH 5.5) with $20 \mathrm{mM}$ ascorbic acid and $20 \mathrm{mM}$ boric acid. Mushroom tyrosinase (Sigma-Aldrich, St. Louis, MO) was then added to the solution to a final concentration of $50 \mathrm{mg} \mathrm{L}^{-1}$. The modification reaction was performed at room temperature for $1 \mathrm{~h}$ under gentle stirring and aeration. DOPA conversion of tyrosine residues was assessed by nitroblue tetrazolium (NBT) staining and amino acid composition analysis as followed in previous studies. ${ }^{23,24}$ The $m$ MAP was freeze dried for further procedures.

\section{NBT staining}

Quinone-carrying quinoproteins and quinonoid substances such as DOPA and DOPA-quinone are detectable by redox- cycling staining in the presence of NBT and glycinate at alkaline $\mathrm{pH}$. After gel electrophoresis of $m \mathrm{MAP}$ which was dissolved in $5 \%(\mathrm{vol} / \mathrm{vol})$ acetic acid, the gel was transferred to a nitrocellulose membrane (Protan BA83; Whatman, GE healthcare, Little Chalfont, UK). Then, the membrane was immersed in $2 \mathrm{M}$ sodium glycinate buffer ( $\mathrm{pH}$ 10) containing $0.5 \mathrm{mg} \mathrm{ml}^{-1} \mathrm{NBT}$ and incubated at room temperature until a blue-purple color developed. The stained membrane was washed with $0.2 \mathrm{M}$ sodium borate solution ( $\mathrm{pH}$ 8.5) and DW.

\section{Amino acid composition analysis}

mMAP was hydrolyzed into amino acids using the ninhydrin reaction. mMAP was placed in a glass vial and dissolved in a $20: 1$ mixture of $6 \mathrm{M} \mathrm{HCl}$ with phenol. The glass vial was sealed after being charged with Argon gas. mMAP was hydrolyzed at $156^{\circ} \mathrm{C}$ for $1 \mathrm{~h}$, then, its hydrolysate was evaporated and washed to remove the solvent. After dissolving the hydrolyzed $m \mathrm{MAP}$ in a dilution buffer, content values of its amino acids including DOPA were determined using an amino acid analyzer (S4300, SYKAM, Germany) and recalculated to find the ratio of DOPA in mMAP.

\section{Preparation of DBBM aggregate using mMAP binder}

DBBM (Ti-Oss; Chiyewon, Guri, Korea) was used as an experimental xenograft bone substitute material for in vivo bone regeneration. mMAP powder was dissolved in endotoxin-free distilled water (HyClone, Logan, UT) to a $300 \mathrm{~g} \mathrm{~L}^{-1}$ concentration. The protein sample was stored in a vacuum-packed state and opened at the time of its use. In total, $10 \mathrm{mg}$ of DBBM particles were mixed with $100 \mu \mathrm{L}$ of $m \mathrm{MAP}$ solution to prepare the DBBM aggregate. To investigate the morphology of osteoblast cells on the DBBM surface, $50 \mu \mathrm{g}$ of $m$ MAP per $\mathrm{cm}^{2}$ of well area was coated to the DBBM particle using the precipitation method as followed in a previous study. ${ }^{21}$ Then, osteoblast MC3T3-E1 cells (RIKEN Cell Bank, Tsukuba, Japan) were seeded on DBBM alone and on $m$ MAP-coated DBBM samples. After 1 day of cultivation under serum-containing conditions, the morphology of the osteoblast cells on the DBBM surfaces was analyzed by scanning electron microscopy (SEM; JSM-6010LV; JEOL Ltd., Tokyo, Japan) using $5 \mathrm{kV}$ secondary electron imaging.

\section{Animal surgery for in vivo bone regeneration}

Fifteen male Sprague-Dawley rats (Orient Bio Inc., Gyeonggido, Korea) weighing $\sim 250-300 \mathrm{~g}$ were housed two per cage in a facility of the specific-pathogen-free-2 (SPF-2) level, which was covered with soft bedding. The rats were allowed free access to food and water in a temperature- and humidity-controlled room $\left(23{ }^{\circ} \mathrm{C}, 50 \%\right)$ with a $12 / 12 \mathrm{~h}$ day/night cycle $(8 \mathrm{am} / 8 \mathrm{pm})$. After a 7 day acclimation, each rat was anesthetized with an intramuscular injection of $30 \mathrm{mg} \mathrm{kg}^{-1}$ Zoletil 50 (tiletamine and zolazepam; Virbac, Carros, France) and $10 \mathrm{mg} \mathrm{kg}^{-1}$ Rompun (xylazine; Bayer, Leverkusen, Germany), and the scalp was incised carefully. Each exposed calvaria was then punctured in a critical size of defect using an $8 \mathrm{~mm}$-diameter trephine bur under sterile saline irrigation, and the hole was filled with $10 \mathrm{mg}$ of Ti-Oss aggregate containing $100 \mu \mathrm{L}$ of mMAP. The 
defect area in the negative-control (sham surgery) group was left empty, whereas that of the DBBM group was filled with only $10 \mathrm{mg}$ of Ti-Oss particles. The incised periosteum and skin were closed in layers with 5-0 Vicryl sutures (Ethicon, Somerville, NJ) after the procedure. After the surgery, the rats were kept in their cages for 8 weeks. To obtain calvarial samples for radiographic and histological analyses, the rats were sacrificed using the formalin-perfusion fixation method.

\section{Radiographic and histological analyses for in vivo bone regeneration}

The rat calvarial specimens were fixed with $10 \%$ formalin for $24 \mathrm{~h}$. Radiographs of the specimens were then taken using X-ray microtomography ( $\mu \mathrm{CT}$ ) (SkyScan 1172; Bruker-microCT, Kontich, Belgium). A total of 683 slices were imaged (10.89 $\mu \mathrm{m}$ per slice for a $590 \mathrm{~ms}$ exposure) for each specimen, and the pictures were analyzed and used to generate a 3D image of $2000 \times 1048$ pixels using CT reconstruction software (NRecon v.1.4.4; Bruker-microCT). The regenerated bone area was quantified using an image analysis program (CTAn v.1.6.0; BrukermicroCT).

After radiographic analysis, the rat calvarial specimens were decalcified in a decal solution (Calci-Clear Rapid; National Diagnostics, Atlanta, GA) for $24 \mathrm{~h}$. The samples were then embedded in paraffin and cut into $3 \mu \mathrm{m}$ sections. The tissue slices were subjected to deparaffinization and hydration steps and then stained with hematoxylin-eosin (H\&E; Sigma-Aldrich), Masson trichrome (MT; Sigma-Aldrich), and toluidine blue (TB; Sigma-Aldrich). Histologic photographs were taken under a BX41 microscope (Olympus, Tokyo, Japan) and analyzed using ToupView software (ToupTek Photonics Co., Hangzhou, China).

\section{In vitro cell attachment and proliferation analyses}

Six- or 24-well polystyrene culture plates (SPL Life Sciences, Pocheon, Korea) were used for the in vitro studies. In total, $50 \mu \mathrm{g}$ of MAP per $\mathrm{cm}^{2}$ of well area was used for surface coating, and the coating method was performed according to a previous study. ${ }^{21}$ A surface coated with PLL (Sigma-Aldrich) was used as a comparative control, and an uncoated, bare surface was used as a negative control. PLL was coated onto culture plates as described in the manufacturer's instructions.

MC3T3-E1 osteoblast cells were maintained in Minimal Essential Medium-alpha ( $\alpha$-MEM; HyClone) supplemented with $10 \%$ (vol/vol) fetal bovine serum (FBS; HyClone) and penicillin/ streptomycin (HyClone) at $37{ }^{\circ} \mathrm{C}$ under $5 \% \mathrm{CO}_{2}$ in a humid atmosphere. The cells were subcultured using 0.05\% trypsinethylenediaminetetraacetic acid (trypsin-EDTA; HyClone) when they reached $80 \%$ confluence. For cell attachment testing, $5 \times$ $10^{4}$ cells ( $>95 \%$ viable) in serum-free medium were pipetted onto each sample-coated 24-well culture plate. The cells were incubated in a humidified incubator $\left(37^{\circ} \mathrm{C}\right.$ and $\left.5 \% \mathrm{CO}_{2}\right)$ for $1 \mathrm{~h}$, allowing them to adhere to the sample-coated culture plates, and unattached or loosely tethered cells were removed from the surfaces using Dulbecco's modified phosphate-buffered saline (DPBS; HyClone). The attached cells on the surface were further cultured in culture medium for $72 \mathrm{~h}$ for a proliferation experiment. A Cell Counting Kit-8 (CCK-8; Dojindo Laboratories, Kumamoto, Japan) was used to quantify cell attachment and proliferation. The CCK-8 assay was performed after the cell attachment experiment and every $24 \mathrm{~h}$ for 3 days. A total of $25 \mu \mathrm{L}$ of the CCK-8 solution was added to each well, and the plates were further incubated for $3 \mathrm{~h}$ at $37^{\circ} \mathrm{C}$. After incubation, the formazan-containing media were transferred to a 96-well plate (SPL Life Sciences) and measured at $450 \mathrm{~nm}$ using a microplate absorbance spectrophotometer (Bio-Rad, Hercules, CA).

\section{In vitro cell spreading and cytoskeletal organization analyses}

MC3T3-E1 cells were cultured in serum-free medium for serum starvation before the cell spreading experiment. In total, $1 \times 10^{5}$ cells in serum-free medium were placed in a sample-coated $12 \mathrm{~mm} \phi$ cover glass (Superior Marienfeld, Lauda-Königshofen, Germany) and incubated for up to $18 \mathrm{~h}$. FITC-conjugated phalloidin (Sigma-Aldrich) and 4',6-diamidino-2-phenylindole (DAPI; Sigma-Aldrich) were used to stain actin filaments and nuclei, respectively. The specimens were analyzed using a fluorescence microscope (Olympus, Tokyo, Japan).

\section{In vitro cell differentiation analyses}

MC3T3-E1 cells were seeded into sample-coated 6-well plates and cultured in the same way as in the proliferation experiment. At $90 \%$ confluence, differentiation of the osteoblast cells was induced by differentiation medium, which was a mixture of 50 $\mu \mathrm{g} \mathrm{mL^{-1 }}$ ascorbic acid, $10 \mathrm{mM}$ sodium phosphate monobasic, and $1 \mathrm{nM}$ dexamethasone (Sigma-Aldrich) in culture medium. The mineralized matrix of the MC3T3-E1 cells at 21 days after differentiation was stained with Alizarin Red S solution (adjusted to $\mathrm{pH} 4.2$ with ammonium hydroxide; Sigma-Aldrich) after formaldehyde fixation. After the removal of the Alizarin Red S solution, the cells were rinsed three times with deionized water. Images of the stained area were obtained using an optical microscope (Olympus).

To evaluate osteogenic gene expression, total RNA was isolated from cells at 7 days after differentiation using the TRIzol® reagent (Invitrogen, Carlsbad, CA) following the manufacturer's protocol. The total RNA was quantified by absorbance readings at $260 \mathrm{~nm}$ using a UV-Vis spectrophotometer (Mecasys, Daejeon, Korea). Following RNA purification, first-strand cDNA was synthesized from $1 \mu \mathrm{g}$ of total RNA using TOPscript ${ }^{\mathrm{TM}}$ RT DryMIX (dT18) (Enzynomics, Daejeon, Korea). Real-time RT-PCR analysis was performed using a thermocycler (LightCycler® 2.0; Roche Applied Science, Basel, Switzerland). Samples $(2 \times$ SYBR Green Mix, $0.5 \mu \mathrm{M}$ each appropriate primer, and cDNA) were amplified in the thermocycler for an initial denaturation at $95{ }^{\circ} \mathrm{C}$ for $15 \mathrm{~min}$, followed by 40 PCR cycles. Each cycle consisted of $95{ }^{\circ} \mathrm{C}$ for $15 \mathrm{~s}, 60{ }^{\circ} \mathrm{C}$ for $30 \mathrm{~s}$, and $72{ }^{\circ} \mathrm{C}$ for $30 \mathrm{~s}$. The oligonucleotide primers for the experiment are shown in Table 1.

The amplified target gene was first detected at a specific fluorescence intensity (Ct, cycle threshold), and data were generated by relative quantification normalized to the housekeeping gene. Briefly, 3 independently tested $\mathrm{Ct}$ values of the 
Table 1 Primers used in the study

\begin{tabular}{lll}
\hline Marker gene & \multicolumn{2}{l}{ Primer sequences } \\
\hline \multirow{2}{*}{ GAPDH } & Forward & $5^{\prime}$-TTGTCTCCTGCGACTTCAACA-3' \\
& Reverse & $5^{\prime}$-GTGGTCCAGGGTTTCTTACTCC-3' \\
Runx2 & Forward & $5^{\prime}$-GACGTGCCCAGGCGTATTTC-3' \\
& Reverse & $5^{\prime}$-AAGTCTGGGGTCCGTCAAGG-3' \\
T1Col & Forward & $5^{\prime}$-GAGCGGAGAGTACTGGATCG-3' \\
& Reverse & $5^{\prime}$-GCTTCTTTTCCTTGGGGTTC-3' \\
BSP & Forward & $5^{\prime}$-TCCATCGAAGAATCAAAGCA-3' \\
& Reverse & $5^{\prime}$-ATGAGCGTGGCCGGTACTTA-3' \\
ALP & Forward & $5^{\prime}$-GCCCTCCAGATCCTGACCAA-3' \\
& Reverse & $5^{\prime}$-GCAGAGCCTGCTTGGCCTTA-3' \\
OCN & Forward & $5^{\prime}$-ACCTCACAGATGCCAAGCCC-3' \\
& Reverse & $5^{\prime}$-TCACTTGTCTGAGGCCGCGT-3'
\end{tabular}

target gene were normalized to those of the glyceraldehyde-3phosphate dehydrogenase (GAPDH) gene for computation of $\Delta \mathrm{Ct}$, and calculation of $\Delta \Delta \mathrm{Ct}$ was then performed by subtracting the $\Delta \mathrm{Ct}$ value of the control group from that of the test group. The fold change in target gene expression in the test group was calculated by the comparative Ct method. ${ }^{25}$ To check for amplification error, a melting-curve analysis (cooling the sample to $65{ }^{\circ} \mathrm{C}$ for $15 \mathrm{~s}$ and then heating slowly to $95{ }^{\circ} \mathrm{C}$, with continuous measurement of fluorescence) was immediately performed at the end of each PCR.

\section{Statistical analysis}

In vivo data were obtained from 3 (for sham surgery) or 6 (for the DBBM-alone and $m$ MAP-bound DBBM groups) individual rats. Independent in vitro experiments were performed at least 3 times, and triplicate samples were analyzed in each experiment. The significance of the data obtained from the control and test groups was statistically analyzed using the one-way analysis of variance (ANOVA) test. A $p$-value of $<0.05$ was considered statistically significant.

\section{Results and discussion}

\section{DOPA modification of MAP and binding of DBBM particles using mMAP}

Due to the absence of post-translational modification machinery in an E. coli system, we performed in vitro DOPA modification using mushroom tyrosinase. Based on the NBT staining, we observed the appearance of a purple band for tyrosinase-treated MAP, whereas no band was observed for the unmodified original sample, indicating successful in vitro DOPA modification (Fig. 1A).

To quantify the DOPA modification yield, we analyzed the amino acid composition of mMAP using a chromatographic method, even though the calculation of the precise yield might have been impossible due to the hydrolysis step during sample preparation. We determined that the in vitro percentage of conversion (modification yield) of tyrosine residues to DOPA molecules was $\sim 38.2 \%$ by amino acid composition analysis (Fig. 1B).
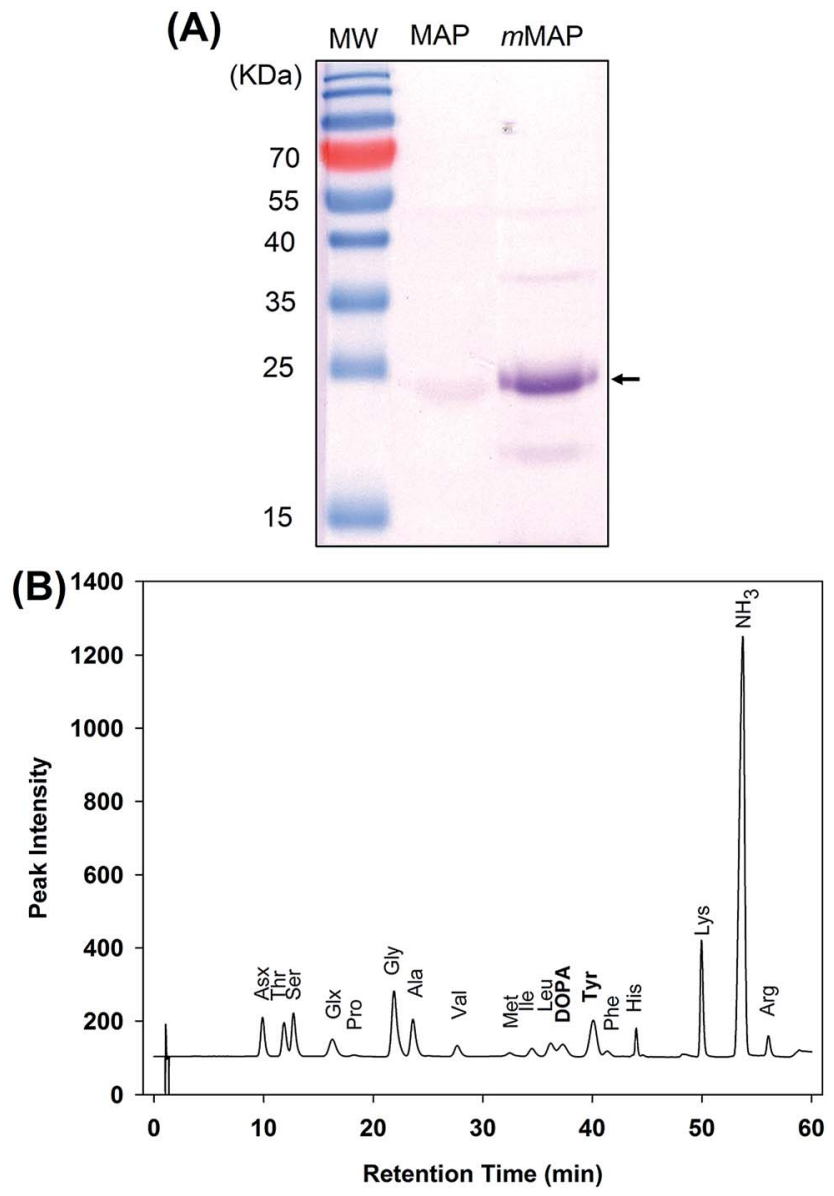

Fig. 1 (A) NBT staining and (B) amino acid composition analyses of recombinant MAP after DOPA modification. Lanes: MW, molecular weight marker; MAP, unmodified MAP; MMAP, DOPA-containing MAP.

To test the binding ability of mMAP in aqueous environments, we prepared the DBBM particles and mMAP to make an aggregate, and compared with the unmodified MAP/DBBM aggregate. We observed that each DBBM particle was easily agglomerated to form a large cluster in the mMAP-treated group (Fig. 2A). Moreover, the DBBM aggregate was not separated into particles when it was lifted by forceps (data not shown), indicating that mMAP effectively binds bone substitute particles. Even though both aggregates of $m \mathrm{MAP} / \mathrm{DBBM}$ and MAP/DBBM had almost a similar appearance in dry environments, the MAP/ DBBM aggregate rapidly dispersed in aqueous environments (Fig. 2A).

Next, prior to the animal experiment, in vitro osteoblast MC3T3-E1 cell adhesion to the mMAP-applied DBBM surface was evaluated. We found that both cell spreading on and the population of the $m \mathrm{MAP}$-applied DBBM were more extensive than on the DBBM alone (Fig. 2B).

\section{In vivo bone regeneration using mMAP-bound DBBM aggregates}

Depending on the applied body parts, in vivo applications often should maintain their performance in aqueous environments such as blood and body fluid. MAP without DOPA modification 

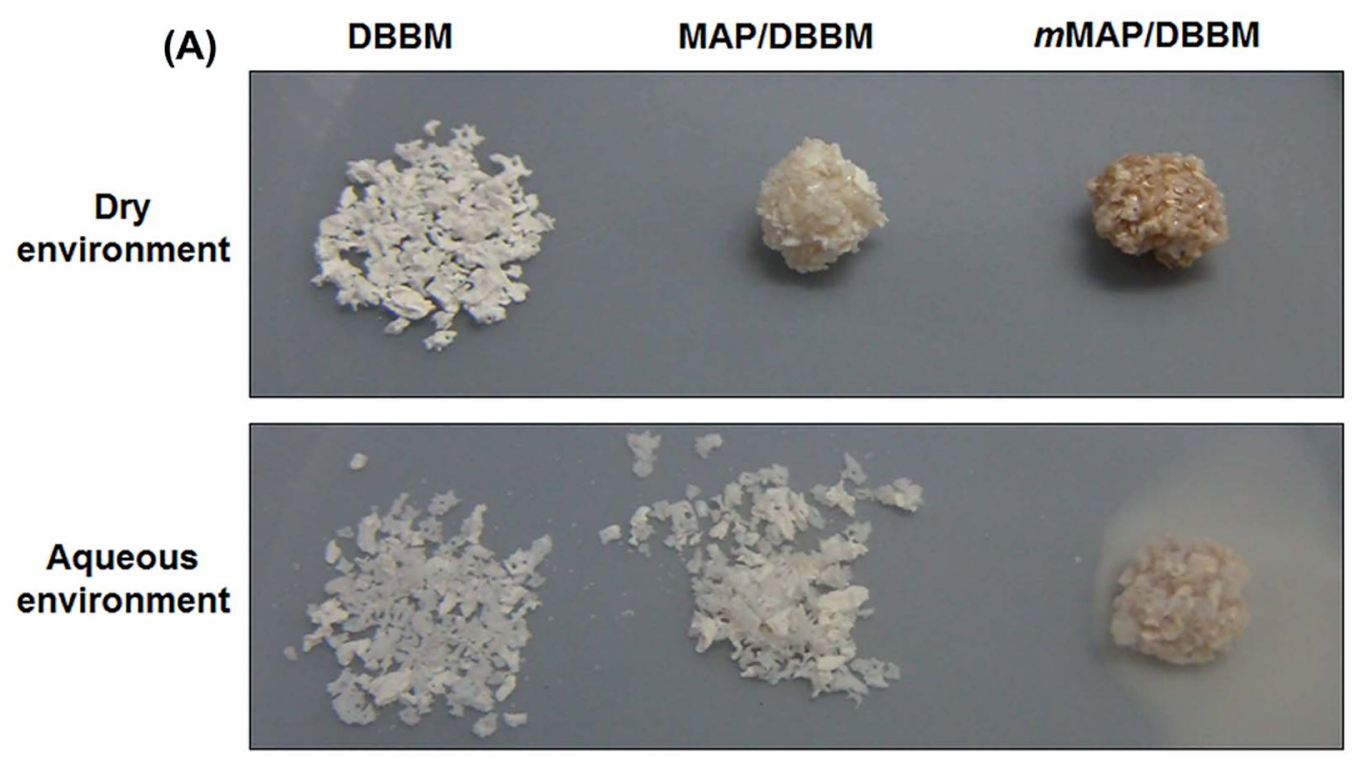

(B)
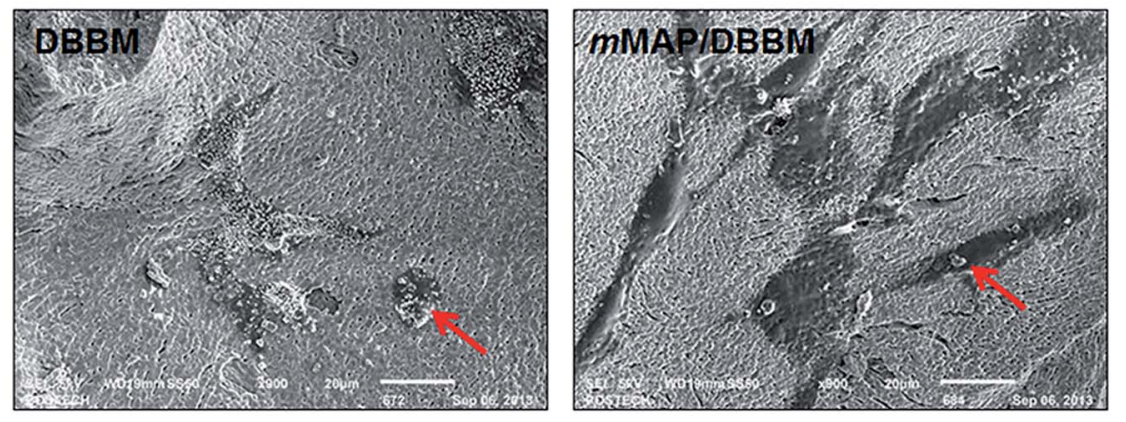

Fig. 2 (A) Photographic images of DBBM particles, MAP-bound DBBM aggregates, and mMAP-bound DBBM aggregates in dry and aqueous environments. (B) Morphology of osteoblast cells on the DBBM particle surface using SEM analysis. The red arrows indicate cells on the DBBM particle surface. The scale bars represent $20 \mu \mathrm{m}$. Abbreviations: DBBM, DBBM alone; MAP/DBBM, MAP-bound DBBM; mMAP/DBBM, mMAPbound DBBM.

may not sustain its adhesive capability during the in vivo regeneration period. Therefore, we conducted in vivo bone regeneration in a critical-sized rat calvarial defect model using the mMAP-bound DBBM aggregate. A sham surgery group was used as a negative control, and a DBBM-alone group was used as a comparative control. The rats were sacrificed after 8 weeks of healing for radiographic and histological analyses. Visualization and quantification of newly formed bone in the criticalsized rat calvarial defect were performed via a $\mu \mathrm{CT}$ system.

Although $\mu \mathrm{CT}$ is powerful technique for the assessment of bone tissue, it is difficult to distinguish a newly formed bone from grafted DBBM due to their compositional similarity. Furthermore, it is known that DBBMs (e.g., Bio-Oss and Ti-Oss) are hardly resorbed during the experimental period. ${ }^{26}$ Therefore, $\mu \mathrm{CT}$ analysis was conducted based on density differences to distinguish the newly formed bone from the grafted DBBM. Images of the transverse plane (Fig. 3A-a) and sagittal plane (Fig. 3A-b) and a 3-dimensional (3D) bone scan (Fig. 3A-c) showed that the defect (white dotted circle in Fig. 3A-a) significantly recovered, forming a new bone, in the DBBM-alone and $m$ MAP-bound DBBM groups.

Whereas the bone defect of the sham surgery group was only $\sim 13 \%$ repaired, the percentage of bone volume per tissue volume in the DBBM-alone group increased by more than $\sim 45 \%$ during the 8 weeks of healing (Fig. 3B). Importantly, the total amount of newly formed bone in the mMAP-bound DBBM group significantly increased, up to $\sim 57 \%$ (Fig. 3B). Notably, we observed a significant loss of DBBM particles from the detect site in the DBBM-alone group (yellow dotted circle in Fig. 3A-a). Therefore, from these radiographic analyses, we could conclude that the superior in vivo bone regeneration effect of $m \mathrm{MAP}$ was attributable to the adhesive nature of MAP, binding DBBM particles in the desired location (the defect site) and possibly recruiting endogenous functional biomolecules to the surface of the DBBM. Furthermore, interestingly, in the rat calvarial defect site, a new bone formed more densely in the mMAPbound DBBM group compared with the DBBM-only group and had a similar density to the normal bone (Fig. 3C). This result might indicate that $m$ MAP has an osteoinductive function in in vivo bone maturation.

After quantification of bone regeneration, we evaluated the quality of the newly formed bone by 3 staining methods: H\&E, MT, and TB staining. Similar to the results of the radiographic analysis, a larger amount of bone formation was clearly observed in the mMAP-bound DBBM group compared with the DBBM-only group by H\&E staining $(40 \times$ magnification; 
(A)

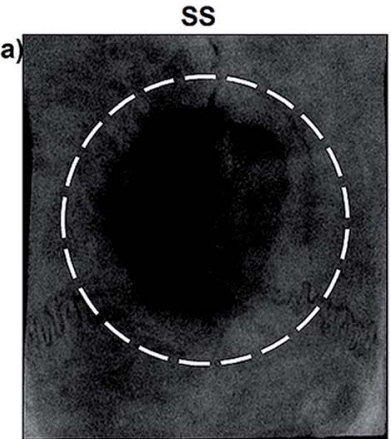

b)

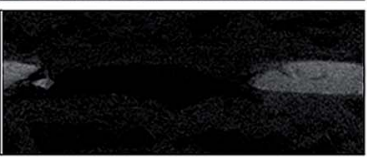

c)

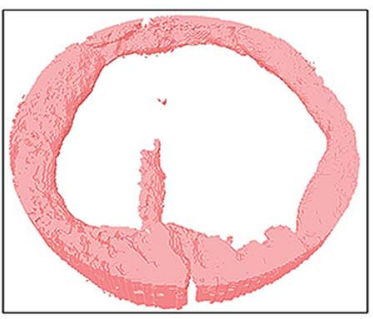

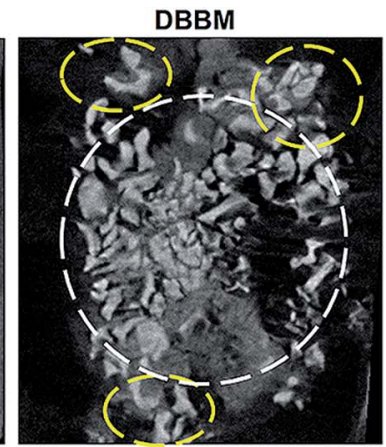
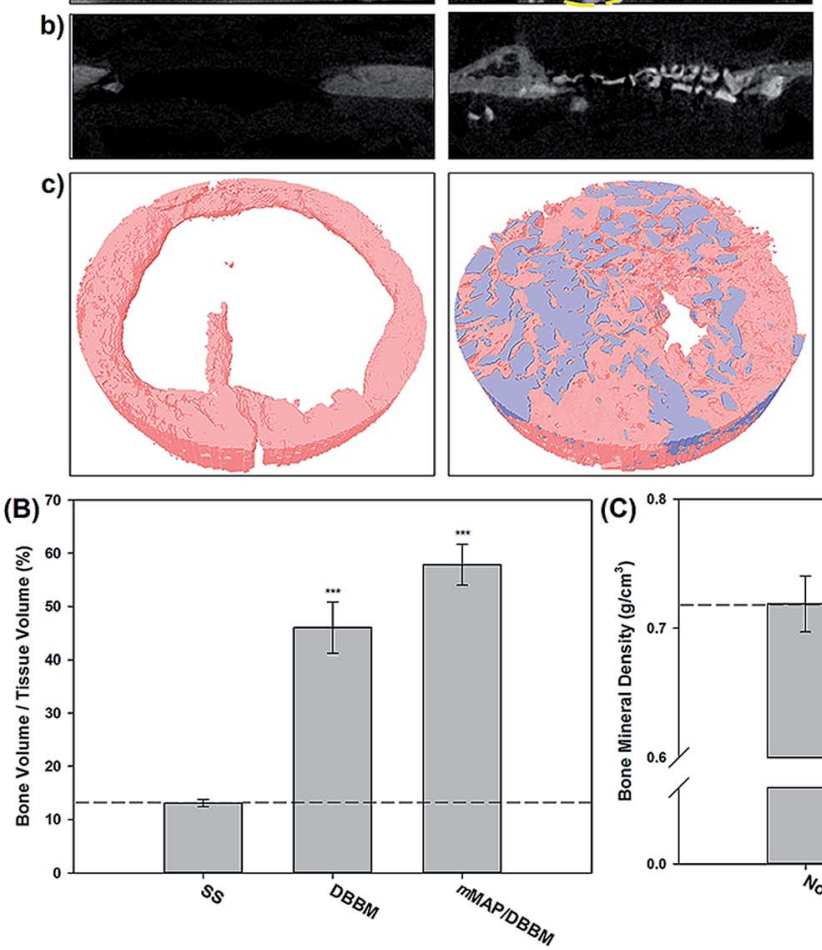

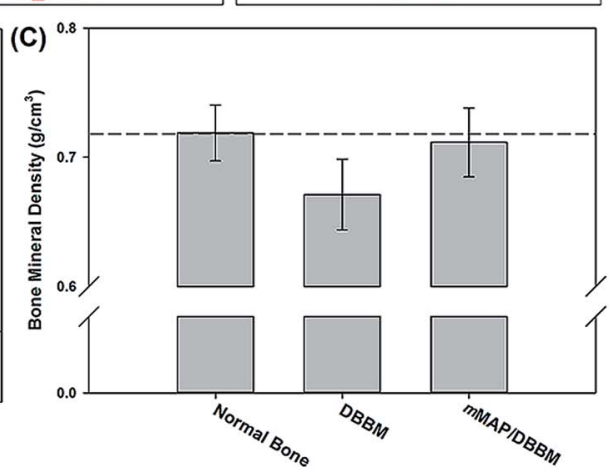

Fig. 3 Microcomputed tomography analyses of bone regeneration in a rat calvarial defect at 8 weeks post-surgery. (A) Images of a) the transverse plane and b) sagittal plane and c) 3D analyses. In the 3D images, the blue color indicates DBBM, the white color indicates empty sites, and the pink color indicates the newly formed bone. (B) Percentage of bone volume per tissue volume and (C) bone mineral density of the defect site. The values and error bars represent the means of six independent animals and standard deviations, respectively, with statistical significance $(* p<0.05, * * p<0.01, * * * p<0.005$, one-way ANOVA). Abbreviations: SS, sham surgery group; DBBM, DBBM-alone group; $m M A P / D B B M$, mMAP-bound DBBM group.

Fig. 4A). In high-resolution images of the MT staining $(100 \times$ magnification; Fig. 4B) and TB staining $(200 \times$ magnification; Fig. 4C), newly formed lamellar bone (yellow arrows) resulting from osteoblastic osteoid formation and osteoid (blue arrows) resulting from osteoblast rimming (white arrows) were detected in the $m$ MAP-bound DBBM group, whereas osteoid from osteoblast rimming was found only adjacent to the DBBM in the DBBM-only group. Furthermore, we observed osteocytes (red arrows) only in the mMAP-bound DBBM group (Fig. 4C). These results indicate that the new bone created between the DBBM particles had already formed before the eighth week of healing and that the bone remodeling process was actively progressing in the $m$ MAP-bound DBBM group.

The terminology of 'osteoinductive activity' was originally defined as the procedure in which osteogenesis is induced. ${ }^{27,28}$ Recently, it is considered that the osteoinduction process is composed of 3 sequential processes; cell/active molecule recruit, cell differentiation, and bone formation. ${ }^{29}$ We suggest that $m$ MAP has osteoinductive activity based on recruitment of cells and active molecules through its unique adhesion ability even though it does not possess autoinduction properties such as BMP-2. Consequently, in vivo bone regeneration was accelerated by $m \mathrm{MAP}$, and subsequently, the bone remodeling process was stimulated in the mMAP-bound DBBM group. Because $m$ MAP can assist bone regeneration in both quantitative and qualitative aspects in the rat calvarial defect model, it could be successfully utilized as a functional biomaterial for bone tissue engineering.

In vitro osteoblast cell behaviors on the $m \mathrm{MAP}$-coated culture surface. Based on the in vivo bone regeneration experiments, we found that $m$ MAP might have osteoinductive ability. Thus, to evaluate the potency of the osteoinductivity of mMAP, we 


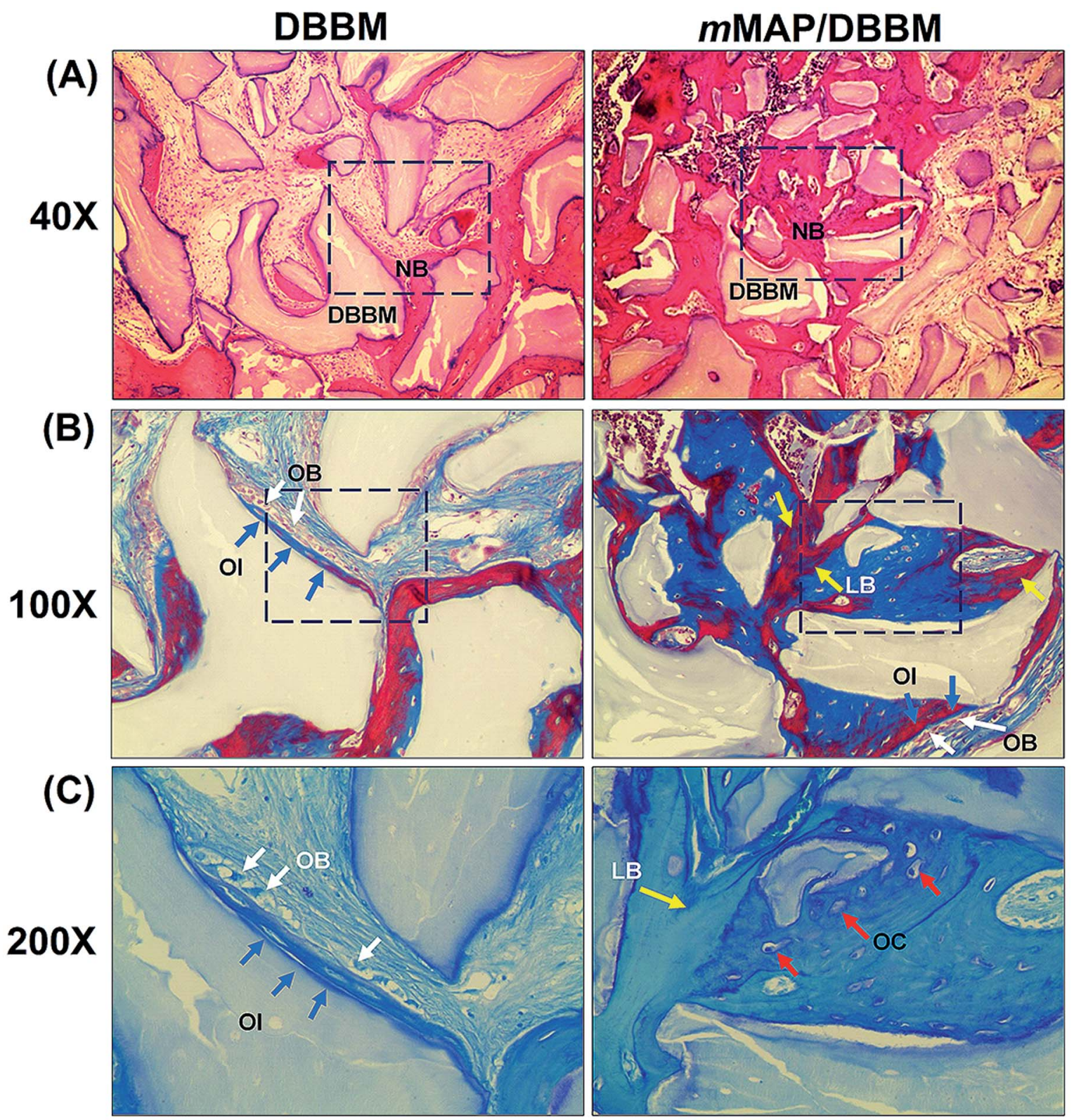

Fig. 4 Histological analyses of bone regeneration in a rat calvarial defect at 8 weeks post-surgery. Images of (A) H\&E staining at $40 \times$ magnification, (B) MT staining at $100 \times$ magnification, and (C) TB staining at $200 \times$ magnification. The white arrows indicate osteoblast cells, the blue arrows indicate osteoid with osteoblastic rimming, the yellow arrows indicate newly formed lamellar bone resulting from osteoblastic osteoid formation, and the red arrows indicate osteocytes. Abbreviations: DBBM, DBBM-alone group; mMAP/DBBM, mMAP-bound DBBM group; NB, newly formed bone; LB, lamellar bone; OB, osteoblast cell; OI, osteoid; OC, osteocyte.

investigated in vitro osteoblast cell behaviors (attachment, proliferation, spreading, and differentiation) on MAP (with or without DOPA)-coated surfaces. An uncoated, bare surface was used as a negative control, and a poly-L-lysine (PLL)-coated surface was used as a positive control because the isoelectric point (pI) ( 9.74) of PLL is similar to the calculated value ( 9.91) for MAP..$^{15}$ The first stage in the process of cell-surface interaction is the cell attachment, which can affect subsequent cellular responses, such as spreading, proliferation, and differentiation. ${ }^{30}$ All cell attachment tests were performed under serum-free conditions to minimize the effects of the diverse adhesion factors in the serum. Although cell attachment was slightly increased on the mMAP-coated surface compared with that of the bare surface, there was no difference from the unmodified MAP-coated surface (Fig. 5A).

Proliferation levels on all sample-coated surfaces showed an at least 2-fold increase compared with the bare surface (Fig. 5B). However, there was also no difference in osteoblast proliferation between the mMAP- and the unmodified MAP-coated surfaces. These results might indicate that the DOPA residues in $m$ MAP do not contribute to cell attachment and proliferation.

Because proliferation levels on all MAP-coated surfaces were increased $(\sim 12 \%)$, regardless of DOPA existence, compared with levels on the PLL-coated surface (Fig. 5B), we can conclude 
that a MAP-coated surface provides a more favorable environment for osteoblast cell proliferation through its adhesive ability and/or accumulation of positively charged ions (e.g., $\left.\mathrm{Ca}^{2+}\right)$ on the coated surface ${ }^{31,32}$ by $\pi$-cation interaction using its rich aromatic amino acid residues. ${ }^{33,34}$

From a morphological perspective, one of the regulating factors for cell fate is the degree of cell spreading. ${ }^{35}$ To examine the effect of $m$ MAP coating on cell spreading, we monitored the morphological changes of MC3T3-E1 cells on the sample-coated surfaces under serum-free conditions using immunocytochemical analysis with fluorescein isothiocyanate (FITC)conjugated phalloidin staining. In low-resolution spreading images, more osteoblast cells were attached on both unmodified MAP- and $m$ MAP-coated surfaces than in the comparative groups (Fig. 5C). Furthermore, we observed that cell spreading on both MAP- and $m$ MAP-coated surfaces was more stretched than spreading in the control groups in high-resolution images (inset image in Fig. 5C). It is known that the cell spreading event typically occurs through interaction of extracellular matrix (ECM) proteins (or their specific peptides) and their receptors. $^{36,37}$ Several studies, however, have demonstrated that osteoblast cell spreading can also be influenced by immobilized calcium ions. ${ }^{38,39}$ Because our experimental samples did not have any activating groups for cell spreading, such as ECM proteins or their specific peptides, we suspect that calcium ions attached to MAP via $\pi$-cation interaction fostered a better spreading environment than in the other control groups under serum-depleted conditions.
After the period of cell proliferation, the ECM of osteoblast cells undergoes compositional and organizational adaptation for mineralization. ${ }^{40}$ Although these changes are typically induced by growth factors and hormones, especially bone morphogenetic protein-2 (BMP-2), the differentiation of osteoblast cells can be also induced in DOPA-contained environments. ${ }^{17-20}$ To evaluate the effect of the DOPA residues in mMAP, we examined the differentiation of MC3T3-E1 cells on samplecoated surfaces. Phenotypic osteoblast differentiation was assessed by measuring the calcium deposition of the ECM of differentiated MC3T3-E1 cells through visualization using Alizarin Red S staining. We observed that the mineral deposition of MC3T3-E1 cells on the mMAP-coated surface was significantly increased compared with deposition on bare and PLL-coated surfaces (Fig. 6A).

Although mineral deposition on the unmodified MAP-coated surface was lower than that on the mMAP-coated surface, this deposition occurred at a much higher level than in the control groups. Thus, similar to previous results on proliferation and spreading, we strongly suggest that the increased calcification was seriously affected by MAP via its adhesive ability and/or electrostatic interaction. Next, genotypic differentiation was determined by the quantification of the expression levels of osteogenic genes, including runt-related transcription factor 2 (Runx2), type 1 collagen (T1Col), bone sialoprotein (BSP), alkaline phosphatase (ALP), and osteocalcin (OCN), using the realtime reverse transcription-polymerase chain reaction (RT-PCR). We found that the expression of all of the transcription factor

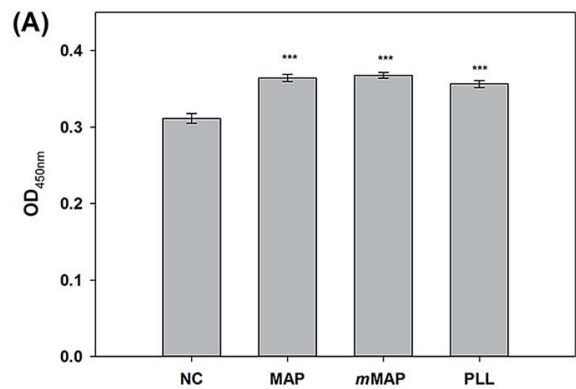

(C)
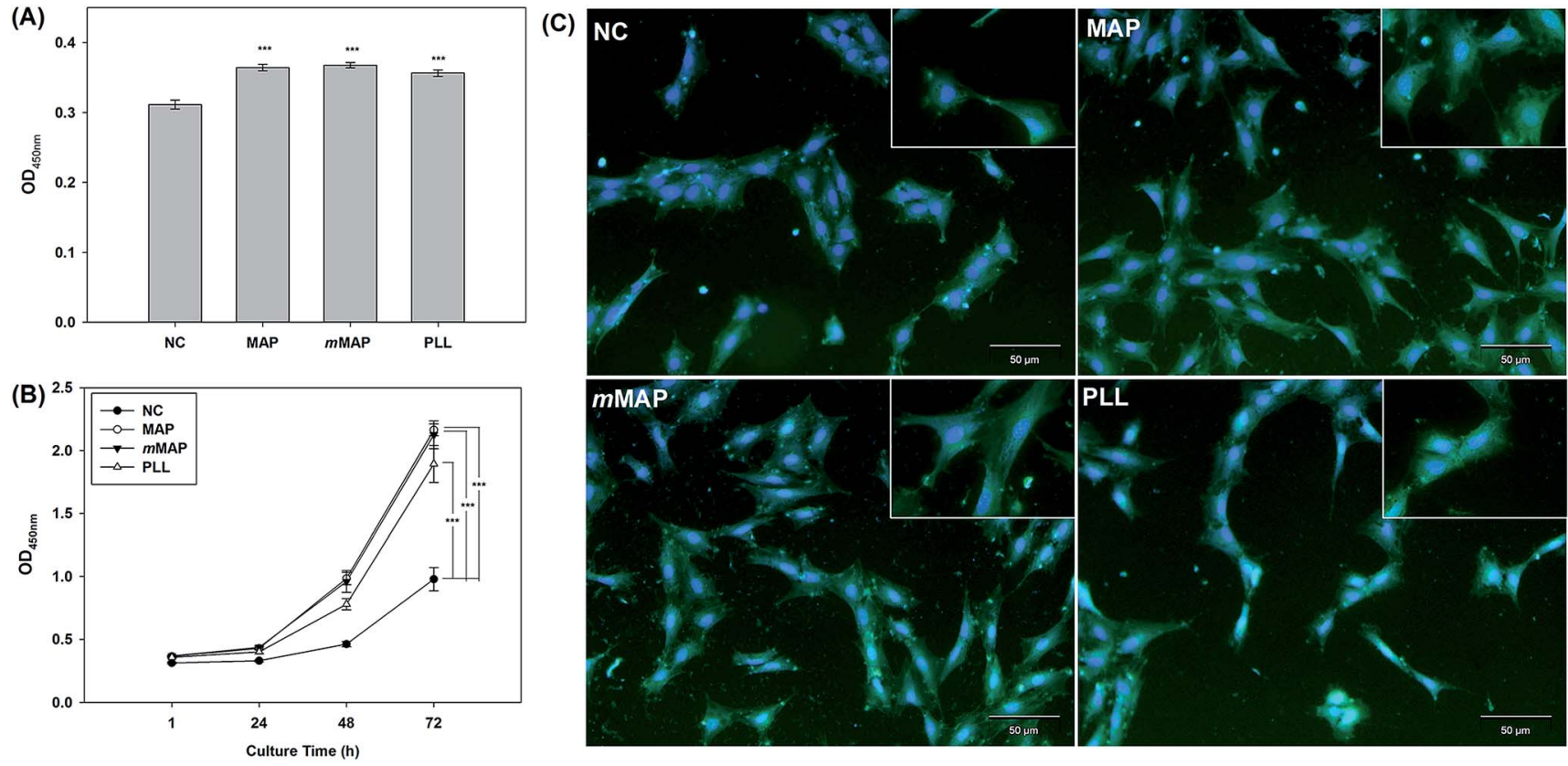

Fig. 5 (A) Attachment, (B) proliferation, and (C) spreading of MC3T3-E1 osteoblast cells on sample-coated surfaces. The concentration of the protein sample used for surface coating was $50 \mu \mathrm{g} \mathrm{cm}^{-2}$. MC3T3-E1 cells were incubated in sample-coated 24-well polystyrene culture plates for $1 \mathrm{~h}$ for cell attachment. The viable cells attached to the surface were measured by a colorimetric assay. The values and error bars represent the means of three independent experiments with triplicate samples and standard deviations, respectively, with statistical significance $(* p<0.05, * * p$ $<0.01, * * * p<0.005$, one-way ANOVA). For the spreading test, cells were added to a cover glass coated with $50 \mu \mathrm{g} \mathrm{cm}{ }^{-2}$ of sample without serum and cultured for $18 \mathrm{~h}$. Actin filaments stained with FITC-phalloidin are presented in green, and nuclei stained with DAPI are blue. The scale bars represent $50 \mu \mathrm{m}$. Abbreviations: NC, bare surface; MAP, unmodified MAP-coated surface; mMAP, DOPA-containing MAP-coated surface; PLL, poly-L-lysine-coated surface. 

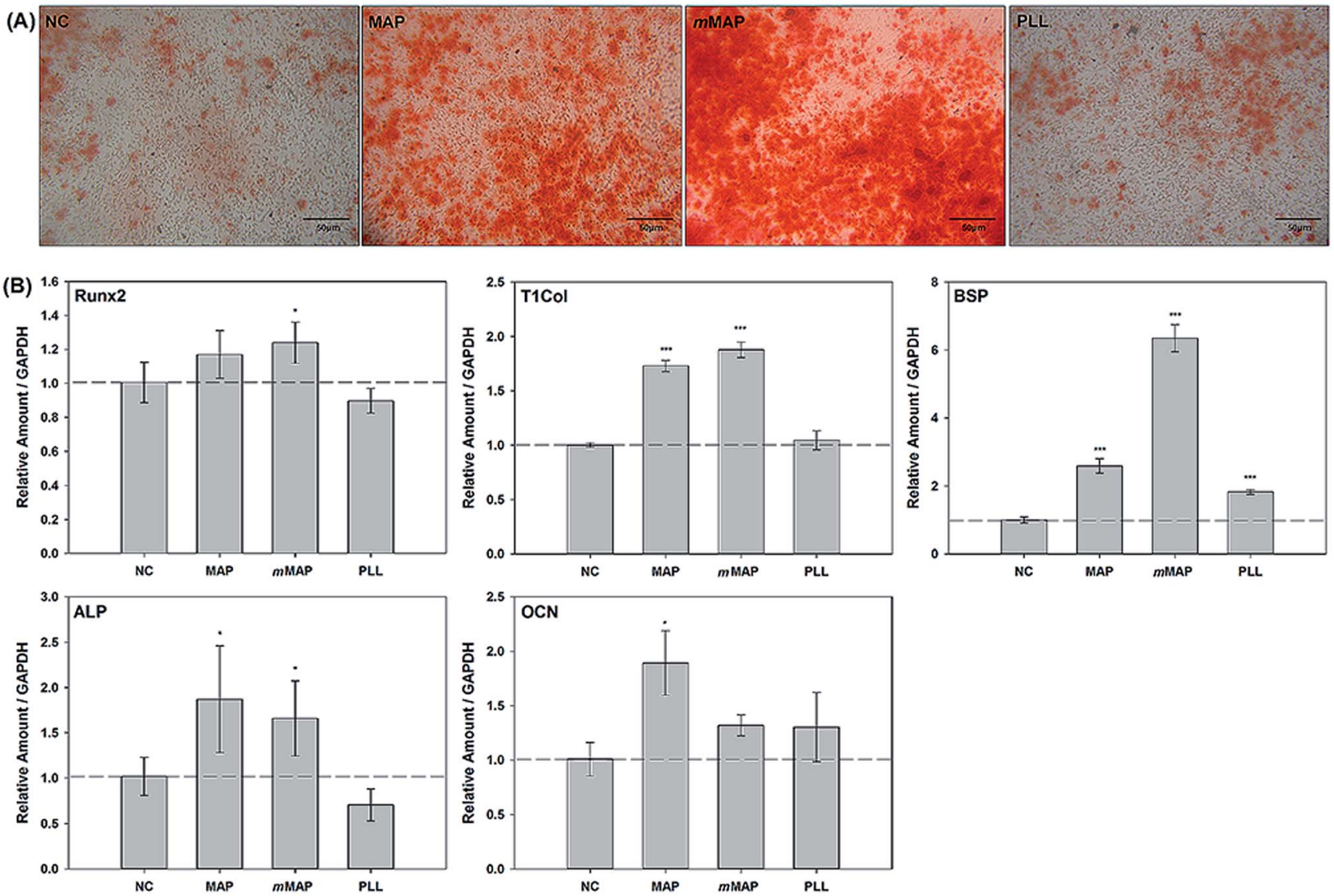

Fig. 6 Differentiation of MC3T3-E1 osteoblast cells on sample-coated surfaces. (A) Matrix mineralization of osteoblast cells on sample-coated 6 -well polystyrene culture plates after 21 days of treatment with differentiation signals, as observed by Alizarin Red S staining. The scale bars represent $50 \mu \mathrm{m}$. (B) The osteogenic gene expression of osteoblast cells was detected by real-time RT-PCR at 7 days after differentiation. The values and error bars represent the means of triplicate samples and standard deviations, respectively, with statistical significance $(* p<0.05$, $* * p<$ $0.01, * * * p<0.005$, one-way ANOVA). Abbreviations: NC, bare surface; MAP, unmodified MAP-coated surface; mMAP, DOPA-containing MAPcoated surface; PLL, poly-L-lysine-coated surface; Runx2, runt-related transcription factor 2; T1Col, type 1 collagen; BSP, bone sialoprotein; ALP, alkaline phosphatase; OCN, osteocalcin.

and marker genes related to osteoblast differentiation was significantly increased in cells on both MAP- and $m$ MAP-coated surfaces compared with a bare surface (Fig. 6B). Interestingly, BSP gene expression was remarkably upregulated on the $m$ MAPcoated surface compared with the unmodified MAP-coated surface. Because it is known that BSP is involved in the mineralization process of osteoblast cells and their maturation, ${ }^{41,42}$ we assumed that the DOPA residues in mMAP are closely related to the maturation of osteoblast cells. From these results, we conclude that osteoblast differentiation can be induced by MAP and that the effect might be further enhanced by the DOPA residues in $m$ MAP.

\section{Conclusions}

Here, we assessed $m \mathrm{MAP}$ as a functional osteoinductive binder of DBBM particles for in vivo bone regeneration. DBBM particles agglomerated to form a large cluster after mixing with a mMAP solution. No loss of grafted DBBM particles was observed in a rat calvarial defect during in vivo healing. New bone formation was significantly improved through grafting of the mMAPbound DBBM aggregate compared with DBBM alone ( $\sim 12 \%$ increase) or the sham surgery control ( $\sim 4 \%$ increase). In addition, a new mature bone that had a similar density to the normal bone was formed. Furthermore, the bone remodeling process was accelerated in the mMAP-bound DBBM group. The in vitro osteogenic differentiation of osteoblast cells on the mMAP-coated surface was upregulated overall compared with differentiation on the unmodified MAP-coated surface. Thus, based on the in vivo and in vitro results, we concluded that the DOPA-containing mMAP has good osteoinductive ability. In conclusion, mMAP can be successfully used as a functional osteoinductive binder for bone substitute-assisted in vivo bone regeneration and could be further utilized in other bone tissue engineering applications.

\section{Acknowledgements}

This work was supported by the Marine Biotechnology Program (Marine BioMaterials Research Center) funded by the Ministry 
of Oceans and Fisheries of Korea (to HJ Cha \& SH Jun), Basic Science Research Program (NRF-2013R1A1A1065373) through the National Research Foundation funded by the Ministry of Science, ICT \& Future Planning of Korea (to SH Jun), and the Rising Star Program funded by POSTECH (to HJ Cha).

\section{References}

1 R. D. Alves, J. A. Demmers, K. Bezstarosti, B. C. Van der Eerden, J. A. Verhaar, M. Eijken, et al., J. Proteome Res., 2011, 10, 4725.

2 J. R. Porter, T. T. Ruckh and K. C. Popat, Biotechnol. Prog., 2009, 25, 1539.

3 A. J. Salgado, O. P. Coutinho and R. L. Reis, Macromol. Biosci., 2004, 4, 743.

4 M. Schieker, H. Seitz, I. Drosse, S. Seitz and W. Mutschler, Eur. J. Trauma, 2006, 32, 114.

5 R. Z. LeGeros, Clin. Orthop. Relat. Res., 2002, 395, 81.

6 A. M. Ambrosio, J. S. Sahota, Y. Khan and C. T. Laurencin, $J$ Biomed Mater Res., 2001, 58, 295.

7 Y. D. Rakhmatia, Y. Ayukawa, A. Furuhashi and K. Koyano, J. Prosthodont. Res., 2013, 57, 3.

8 J. M. Song, S. H. Shin, Y. D. Kim, J. Y. Lee, Y. J. Baek, S. Y. Yoon, et al., Int. J. Oral Sci., 2014, 6, 87.

9 J. Schmid, C. H. Hämmerle, A. J. Olah and N. P. Lang, Clin. Oral Implants Res., 1994, 5, 125.

10 A. S. Monteiro, L. G. Macedo, N. L. Macedo and I. Balducci, Med. Oral Patol. Oral Cir. Bucal, 2010, 15, e401.

11 A. Baldini, D. Zaffe and G. Nicolini, Ann. Stomatol., 2010, 1, 2.

12 Z. Schwartz, M. Goldstein, E. Raviv, A. Hirsch, D. M. Ranly and B. D. Boyan, Clin. Oral Implants Res., 2007, 18, 204.

13 H. G. Silverman and F. F. Roberto, Mar. Biotechnol., 2007, 9, 661.

14 H. J. Cha, D. S. Hwang and S. Lim, Biotechnol. J., 2008, 3, 631.

15 D. S. Hwang, Y. Gim, H. J. Yoo and H. J. Cha, Biomaterials, 2007, 28, 3560.

16 M. J. Sever, J. T. Weisser, J. Monahan, S. Srinivasan and J. J. Wilker, Angew. Chem., Int. Ed., 2004, 43, 448.

17 W. G. La, J. Y. Shin, S. H. Bhang, M. Jin, H. H. Yoon, S. S. Noh, et al., Tissue Eng., Part A, 2013, 19, 1255.

18 Y. T. Liu, T. M. Lee and T. S. Lui, Colloids Surf., B, 2013, 106, 37.
19 S. H. Kim, J. K. Park, J. H. Hong, H. S. Jung, K. S. Hong, J. H. Lee, et al., J. Biomater. Appl., 2012, 27, 143.

20 G. T. Jones, X. C. Jian and D. M. Laskin, J. Oral Maxillofac. Surg., 1996, 54, 470.

21 B.-H. Choi, Y. S. Choi, D. G. Kang, B. J. Kim, Y. H. Song and H. J. Cha, Biomaterials, 2010, 31, 8980.

22 B. J. Kim, Y. S. Choi and H. J. Cha, Angew. Chem., Int. Ed., 2012, 51, 675.

23 S. Lim, Y. S. Choi, D. G. Kang, Y. H. Song and H. J. Cha, Biomaterials, 2010, 31, 3715.

24 B. Yang, D. G. Kang, J. H. Seo, Y. S. Choi and H. J. Cha, Biofouling, 2013, 29, 483.

25 K. J. Livak and T. D. Schmittgen, Methods, 2001, 25, 402.

26 A. K. Schlegel and K. Donath, J. Long-Term Eff. Med. Implants, 1998, 8, 201.

27 M. R. Urist, B. F. Silverman, K. Buring, F. L. Dubuc and J. M. Rosenberg, Clin. Orthop. Relat. Res., 1967, 53, 243.

28 T. Albrektsson and C. Johansson, Eur. Spine J., 2001, 10, S96.

29 R. J. Miron and Y. F. Zhang, Crit. Rev. Oral Biol. Med., 2012, 91, 736.

30 R. G. LeBaron and K. A. Athanasiou, Tissue Eng., 2000, 6, 85. 31 G. Mendonça, D. B. Mendonça, F. J. Aragão and L. F. Cooper, Biomaterials, 2008, 29, 3822.

32 M. Ohgaki, T. Kizuki, M. Katsura and K. Yamashita, J. Biomed. Mater. Res., 2001, 57, 366.

33 D. S. Hwang, H. Zeng, Q. Lu, J. Israelachvili and J. H. Waite, Soft Matter, 2012, 8, 5640.

34 Q. Lu, D. X. Oh, Y. Lee, Y. Jho, D. S. Hwang and H. Zeng, Angew. Chem., Int. Ed., 2013, 52, 3944.

35 C. S. Chen, M. Mrksich, S. Huang, G. M. Whitesides and D. E. Ingber, Science, 1997, 276, 1425.

36 B. M. Gumbiner, Cell, 1996, 84, 345.

37 O. Drevelle, E. Bergeron, H. Senta, M. A. Lauzon, S. Roux, G. Grenier, et al., Biomaterials, 2010, 31, 6468.

38 C. A. O'Neill and C. S. Galasko, Calcif. Tissue Int., 2000, 67, 53.

39 A. Bigi, B. Bracci, F. Cuisinier, R. Elkaim, M. Fini, I. Mayer, et al., Biomaterials, 2005, 26, 2381.

40 J. B. Lian and G. S. Stein, Iowa Orthop. J., 1995, 15, 118.

41 G. S. Stein, J. B. Lian and T. A. Owen, FASEB J., 1990, 4, 3111. 42 J. T. Stubbs III, K. P. Mintz, E. D. Eanes, D. A. Torchia and L. W. Fisher, J. Bone Miner. Res., 1997, 12, 1210. 